\title{
Electrospun Polymer Nanomaterials: Preparation, Characterization, and Application
}

\author{
Aihua He, ${ }^{1}$ Ruigang Liu, ${ }^{2}$ Huarong Nie, ${ }^{3}$ and Yaqin Huang ${ }^{4}$ \\ ${ }^{1}$ Key Laboratory of Rubber-Plastics, Ministry of Education/Shandong Provincial Key Laboratory of Rubber-Plastics, \\ School of Polymer Science and Engineering, Qingdao University of Science and Technology, Qingdao, Shandong 266042, China \\ ${ }^{2}$ State Key Laboratory of Polymer Physics and Chemistry, Beijing National Laboratory of Molecular Sciences, Institute of Chemistry, \\ Chinese Academy of Sciences, Beijing 100190, China \\ ${ }^{3}$ Department of Bioengineering, University of Washington, Seattle, WA 98195, USA \\ ${ }^{4}$ Beijing Laboratory of Biomedical Materials, Beijing University of Chemical Technology, Beijing 10029, China
}

Correspondence should be addressed to Aihua He; aihuahe@iccas.ac.cn

Received 12 July 2014; Accepted 12 July 2014; Published 26 August 2014

Copyright (C) 2014 Aihua He et al. This is an open access article distributed under the Creative Commons Attribution License, which permits unrestricted use, distribution, and reproduction in any medium, provided the original work is properly cited.

Electrospinning is a facile technology for the fabrication of nanostructured materials. With the varied solution properties and electrospun parameters, different nanostructures can be achieved for specific purpose. Up to now, a large amount of polymers has been electrospun for numerous potential applications including biomedical tissue scaffolds, drugs delivery, and semiconductor nanowire synthesis. To put forward the development of this technique and the applications of electrospun nanomaterials, a number of important issues have been addressed such as the fabrication and applications of electrospun nanofibers, advances in electrospun functional nanofibers, and electrospun nanoporous materials. In this special issue, we have invited ten papers that address such issues.

One of the papers of this special issue describes the fabrication of diclofenac sodium (DS) loaded Eudragit L100 nanofibers by a modified coaxial electrospinning for colontargeted sustained release, which helps to avoid the clogging problem of spinneret in electrospinning. Another paper presents an interesting attempt to use PCL nanofiber containing marine seaweeds-extracellular polymeric substance for neural tissue repair. Another paper is about the technology of orderly printing of microdroplets by means of electrohydrodynamic print (EHDP) with alternating current, which would accelerate the application of inkjet printing in the field of micro-/nanosystem production.
One of the papers addresses the introduction of CNTs in aligned electrospun polycaprolactone/gelatin nanofibrous matrices as the growth environments of Schwann cells for peripheral nerve repair. The effects of diameters, alignment, and the content of CNTs in the nanofibers on cell behaviors are discussed. Another paper describes direct write of orderly micro-/nanofibrous structure by electrohydrodynamic system on insulate substrate, which is an interesting attempt to promote the application of EDW technology on the flexible electronics. Another paper simulates a novel electrospinning method, crater-like electrospinning, by two-phase computational fluid dynamics for a deep understanding of two-phase flow and microfluid jets production in electrospinning process. Another paper presents tunable structure of electrospun regenerated silk fibroin mats by annealing in water vapor for the improved mechanical properties, which is contributed to the extension of posttreatment of electrospun matrix.

One of the papers proposes using two opposite emitting electrode electrospinning setups to get naproxen (NAP)/cellulose acetate hybrid nanofibers. The distribution and release behavior of the drugs in the nanofibers are dependent on the electrode polarity. Another paper illustrates the fabrication of ultrafine carbon fibers with a nanoporous structure by the template method based on the electrospun of silica nanoparticles (NPs) embedded in PVA nanofibers, thermal annealing, chemical vapor deposition, and 
alkali treatment, which provides an indication of fabricating inorganic nanofibers by electrospinning polymer nanofibers. Another paper addresses the effect of surfactants on the diameter and morphology of electrospun nanofibers, which shows us a new route to increase the uniformity of electrospun nanofibers and thereby the industrial applications.

Aihua He Ruigang Liu Huarong Nie Yaqin Huang 

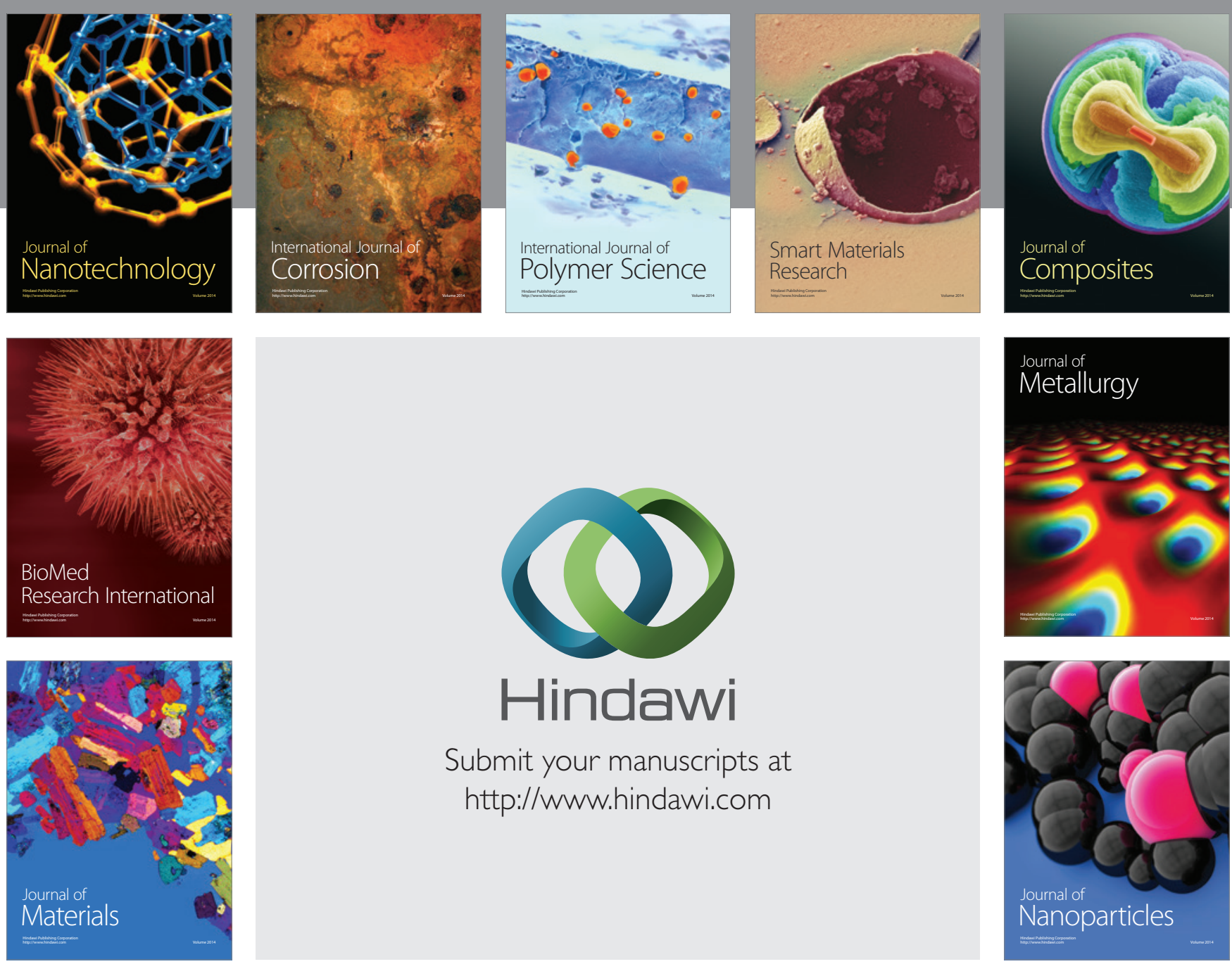

Submit your manuscripts at http://www.hindawi.com
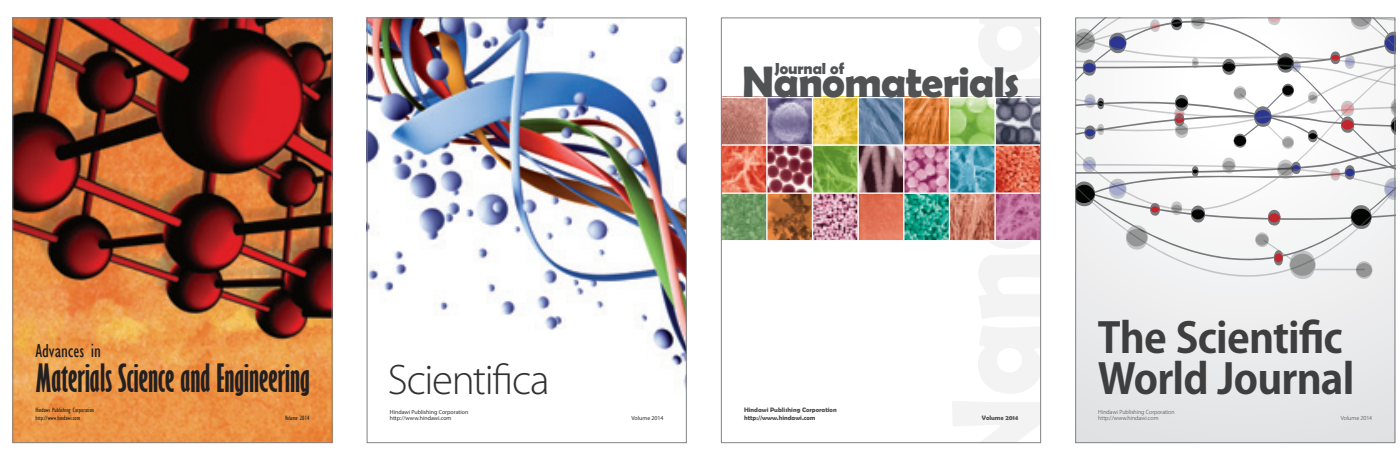

\section{The Scientific World Journal}
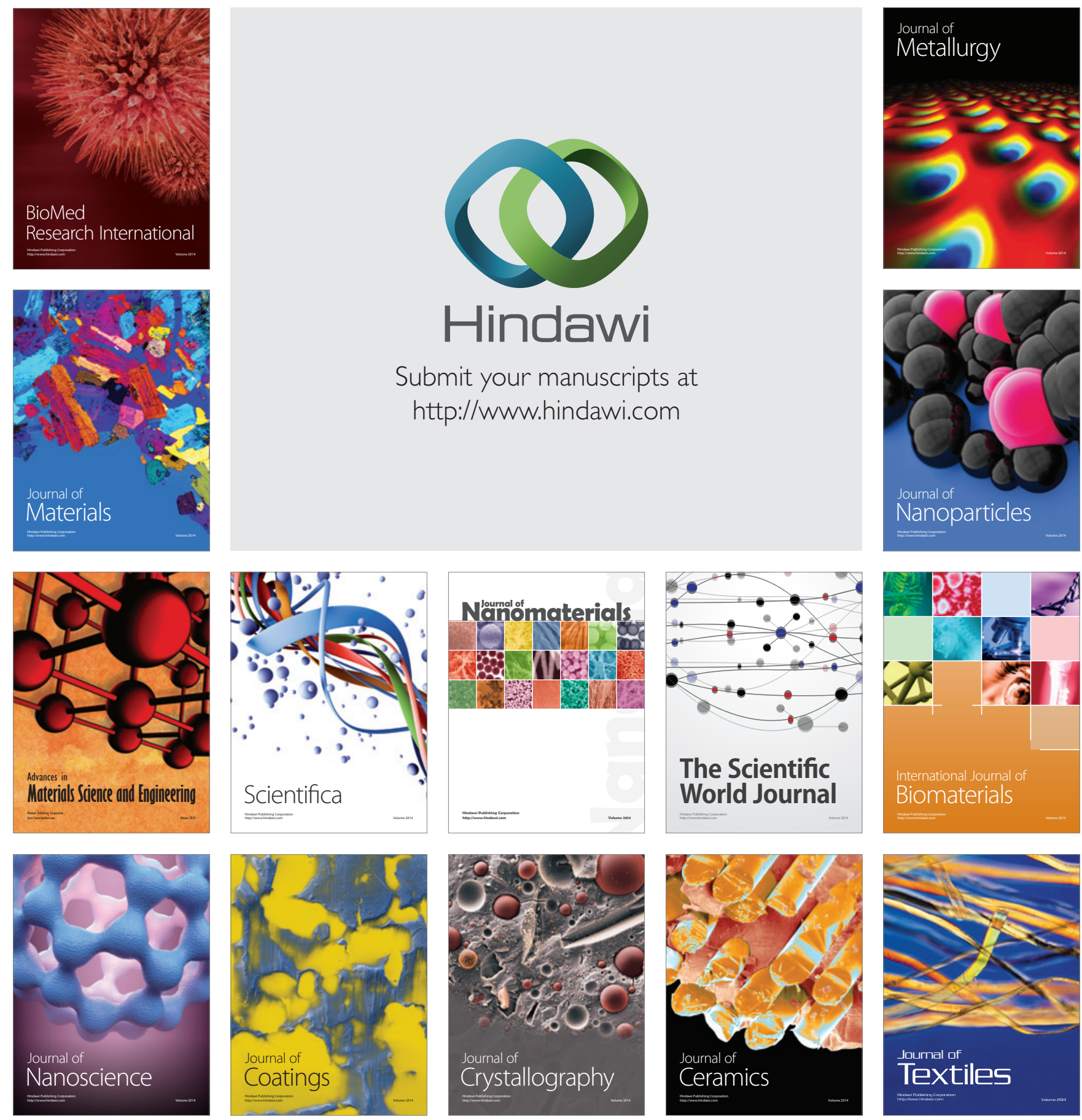